NEUES AUS DER INDUSTRIE

\section{3rd Austrian Symposium on Carbon Based Coatings (CBC 2014)}

Am 4. und 5. Juni 2014 treffen sich Vertreter aus Forschung, Entwicklung und Industrie auf Schloss Seggau (Steiermark - Österreich), um den aktuellen Stand der Technik und die neuesten Entwicklungstrends auf dem Gebiet der Herstellung, Entwicklung, Charakterisierung und Anwendung von Kohlenstoffbasis-Schichten (Diamantschichten, amorphe Kohlenstoffschichten, DLC-Schichten (diamond-like carbon), Graphen) vorzustellen und zu diskutieren. Für die Impulsreferate konnten hochkarätige internationale Experten gewonnen werden:

- Prof. Dr. John Robertson: Properties and Applications of Diamondlike Amorphous Carbons, University Cambridge (United Kingdom)

- Dr. Bernhard C. Bayer: Graphene: Fundamentals, Synthesis and Applications, University of Cambridge (United Kingdom)

- Prof. Dr. Esteban Broitman: Tribology of Carbon-based Coatings: Recent Achievements and Perspectives, Linköping University, (Sweden)
Das chemische Kohlenstoff mit der Ordnungszahl 6 kommt in der Natur in gediegener (reiner) Form (Diamant, Graphit) und chemisch gebunden (z. B. in Form von Carbonaten, Kohlenstoffdioxid, Erdöl, Erdgas und Kohle) vor. Aufgrund seiner besonderen Elektronenkonfiguration besitzt es die Fähigkeit zur Bildung komplexer Moleküle und weist von allen chemischen Elementen die größte Vielfalt an chemischen Verbindungen auf. Kohlenstoffverbindungen bilden auch die molekulare Grundlage das Leben auf unserer Erde.

Im Werkstoff Stahl führen kleine Anteile von Kohlenstoff im Eisen zu den typischen und für die technische Anwendung relevanten Eigenschaften (Festigkeit, Streckgrenze, Härte, Härtbarkeit). Auch in den Kunststoffen ist das Element $C$ ein wesentlicher Bestandteil, der aber auch in keramischen Stoffen, wie Titancarbid, Wolframcarbid (wichtiger Bestandteil des Hartmetalls), Siliziumcarbid, Chromcarbid enthalten ist. Die reinen Kohlenstoffmodifikationen Graphit und Diamant finden technische Anwendung in der Werkzeugtechnik, Metallurgie, Elektronik, Schmuckindustrie, etc.

Kohlenstoff- und Kohlenstoffbasisschichten mit Dicken von wenigen Nanometern bis einige 100 Mikrometer finden heute Anwendungen als Niedrigreibungsschichten, Verschleißschutzschichten, elektrische Isola- tionsschichten, Widerstandsschichten, Gasbarriereschichten, biokompatible Beschichtungen, dekorative Schichten, Anti-Sticking-Schichten, optische Sperrschichten und mehr. Ultradünnen Schichten, die nur aus einer Atomlage Graphit bestehen und als Graphen bezeichnet werden, werden zukünftig vielfältigste Anwendungspotentiale vorhergesagt. Aber auch bei den etablierten Diamantschichten und DLC-Schichten sind zukünftig noch interessante Entwicklungen und neue Anwendungen zu erwarten.

Nähere Informationen zur Veranstaltung finden Sie unter:

www.asmet.at/cbc2014

Das wissenschaftliche Programm des Symposiums sieht auch eine Exkursion zum Institut für Oberflächentechnologien und Photonik der JOANNEUM RESEARCH (Weiz) und zum Zentrum für Elektronenmikroskopie (Graz) vor.

Wenn Sie selbst einen Vortrag im Umfang von 20 min halten möchten, bitten wir um Übermittlung eines Abstracts und Ihre Online-Anmeldung.

Die Veranstalter The Austrian Society for Metallurgy and Materials (ASMET), JOANNEUM RESEARCH Forschungsgesellschaft $\mathrm{mbH}$, Materials Center Leoben Forschung $\mathrm{GmbH}$ (MCL) und die Montanuniversität Leoben (MUL) freuen sich über Ihr Mitwirken und/oder über Ihre Teilnahme an dem Symposium. 